\title{
Sniffing out disease
}

Since the first discovery of the odorant receptors, several families of receptors that detect odorants or pheromones have been identified. Rodriguez and colleagues now report the discovery of a new class of chemosensory receptor and show that these receptors can detect disease-related molecules.

Many animals detect pheromones and other social cues through

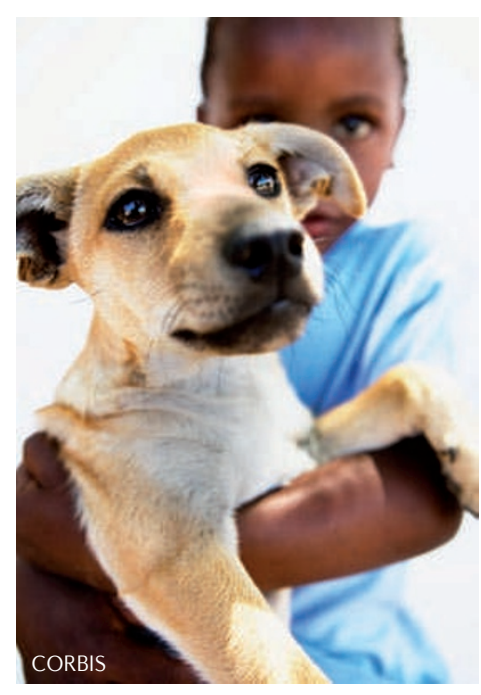

sensory neurons in the vomeronasal organ (VNO). However, the known vomeronasal receptors, V1R and $\mathrm{V} 2 \mathrm{R}$, are not expressed in every vomeronasal neuron, suggesting the existence of additional vomeronasal receptors. The authors therefore examined the expression of putative receptors in the VNO and found that five members of the formyl peptide receptor (FPR) gene family ( Fpr-rs 1 (also known as Fpr3), Fpr-rs3, Fpr-rs4, Fpr-rs6 and Fpr-rs7) are expressed in mouse, rat and gerbil vomeronasal neurons. Using double in situ hybridizations, the authors showed that individual neurons expressed only one of the five transcripts and, furthermore, that there was no co-expression with V1R or V2R. Such a monogenic transcription pattern is characteristic of all the olfactory receptor families identified to date.

Other members of the FPR family are expressed by immune cells and recognize molecules associated with disease, such as peptides released by bacteria or during inflammation. To investigate whether the vomeronasal
FPR proteins also respond to these ligands, the authors used a $\mathrm{Ca}^{2+}$ dye to detect changes in neuronal activity in cultured vomeronasal neurons and whole-mount preparations of the VNO in response to four disease-related ligands. 4-10\% of vomeronasal neurons responded to at least one of the ligands tested: some cells responded exclusively to one particular ligand, whereas others responded to two ligands.

These findings suggest that the VNO can detect molecules related to disease or inflammation. How this information is used is unknown; however, the authors speculate that it could help animals to avoid rotten food or identify unhealthy members of their own species in order to aid effective social interactions. Further work may investigate these hypotheses as well as identify additional ligands for this receptor family.

\section{Katherine Whalley}

ORIGINAL RESEARCH PAPER Rivière, S. et al.

Formyl peptide receptor-like proteins are a novel family of vomeronasal chemosensors. Nature 22 Apr 2009 (doi:10.1038/nature08029) 